\title{
Liver fibrosis in a patient with familial homozygous hypobetalipoproteinaemia: possible role of vitamin supplementation
}

\author{
J-Y Scoazec, M-E Bouma, J-F Roche, D Blache, N Verthier, G Feldmann, G Gay
}

\begin{abstract}
A case of apolipoprotein B-related disorder is reported in which liver fibrosis developed without long term administration of medium chain triglycerides, previously incriminated in the pathogenesis of this lesion. The patient was a young woman in whom the diagnosis of familial homozygous hypobetalipoproteinaemia was made at the age of 21 . A first liver specimen taken at diagnosis revealed steatosis, hypertrophic Golgi apparatus and proliferating smooth endoplasmic reticulum. The patient was treated with vitamin $A$ and $E$ supplementation only. Two years later, a second liver biopsy, carried out because of increased serum alanine aminotransferase concentrations, showed fibrosis, mild cytolysis and marked mitochondrial alterations. Hepatic level of vitamin A was increased. This finding supports the hypothesis that liver disease observed in our patient might be an adverse effect of vitamin supplementation. Our observation underlines the importance of including liver function tests in the follow up of patients with apolipoprotein B-related disorders.
\end{abstract}

Familial homozygous hypobetalipoproteinaemia belongs to a heterogeneous group of inherited disorders in which alterations in the expression of apolipoprotein B (apoB) result in abnormalities of lipid transport and metabolism. ${ }^{12}$ Familial homozygous hypobetalipoproteinaemia is an autosomal dominant disorder, caused by a mutation or deletion in the apoB gene. ${ }^{34}$ Laboratory examination shows: (a) low plasma concentrations of cholesterol and triglycerides, (b) absence of chylomicrons, very low and low density lipoproteins in the plasma, (c) absence of detectable plasma concentrations of the two forms of apolipoprotein B, that is, apoB-100, synthesised by the liver, and apoB-48, originating from the intestine. Familial homozygous hypobetalipoproteinaemia is clinically and biochemically similar to abetalipoproteinaemia, which differs by its recessive mode of transmission and by the absence of detectable structural abnormalities in the apoB gene. ${ }^{56}$

The prognosis of those two apoB-related disorders mainly depends on the consequences of the malabsorption syndrome and on progressive neurological alterations, caused by a deficiency in the lipid soluble vitamin E. ${ }^{78}$ Additional complications, however, in the form of liver fibrosis and/or cirrhosis, have been described in some cases of apoB-related disorders, all reported as abetalipoproteinaemia..$^{9-11}$ The relationship of liver disease with the lipoprotein disorder, if any, remained obscure. A possible toxic effect of the medium chain triglycerides included in the low fat diet recommended to the patients has been suggested. ${ }^{9}$ The use of such compounds in the treatment of apoB-related disorders has, therefore, been questioned, despite their beneficial role in malabsorption and steatorrhoea.

We were therefore prompted to report the observation of a patient with familial homozygous hypobetalipoproteinaemia in whom liver fibrosis appeared in the absence of long term administration of medium chain triglycerides, in order to: (a) further illustrate the possible occurrence of severe liver disease in apoB-related disorders, (b) gain some insight into the pathogenesis of hepatic lesions, and (c) underline their possible relevance to the management of these patients.

\section{Patient history}

The patient is a young Caucasian woman, CD, born on 30 January, 1965. From 1984, she experienced chronic fatty diarrhoea, for which she was referred to the Centre Hospitalier de Verdun in 1986, at the age of 21 . She measured $150 \mathrm{~cm}$ and weighed $38 \mathrm{~kg}$. Clinical examination showed no other abnormality. Hormonal status was normal. Peripheral blood examination disclosed marked acanthocytosis. Laboratory examination showed: (a) marked hypocholesterolaemia $(0 \cdot 19 \mathrm{~g} / 1$; normal range: $1 \cdot 5-2)$ and hypotriglyceridaemia $(0.06 \mathrm{~g} / \mathrm{l}$; normal range: 0.5-1.35), (b) absence of detectable serum concentrations of apoB (I Beucler, Service de Biochimie, Hôpital Saint-Antoine, Paris). ${ }^{12}$ Gastrointestinal endoscopy showed diffuse snowy colouration of the duodenum and jejunum. Jejunal and ileal biopsies obtained by enteroscopy and ileoscopy disclosed heavy accumulation of fat vacuoles within enterocytes; no villous atrophy was found. Familial studies revealed half-reduced plasma concentrations of cholesterol, triglycerides, and apoB in the mother, sister, and brother, whose clinical examination was normal. ${ }^{12}$ The diagnosis of familial homozygous hypobetalipoproteinaemia was therefore raised.

At the time of diagnosis, liver tests were normal. Computerised tomography suggested diffuse fatty infiltration and liver biopsy was taken. Histological examination showed macrovesicular steatosis, predominating in centrilobular areas and present in about $30 \%$ of the hepatocytes. No fibrosis was visible. There was no cytolysis and no inflammatory infiltrate. Ultrastructural examination showed a spectrum of 


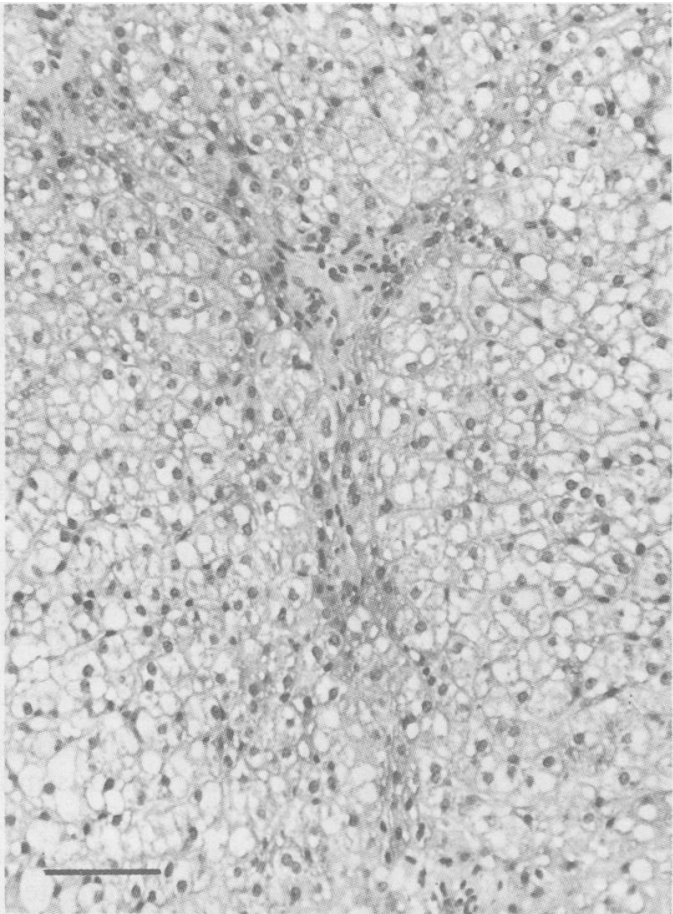

Figure 1: Histological aspect of the second liver specimen. At this low magnification, fibrosis and massive steatosis are evident. (Light microscopy; Gomori's method for demonstration of reticulin fibres). Bar $=10 \mu \mathrm{m}$.

hepatocytic changes, associating: (a) steatosis, (b) mild mitochondrial changes, including the presence of paracrystalline inclusions in a few organelles, (c) proliferation of smooth endoplasmic reticulum, and (d) hypertrophy of Golgi apparatus, devoid of lipoprotein like structures. At this time, serum vitamin $\mathrm{E}$ concentration was

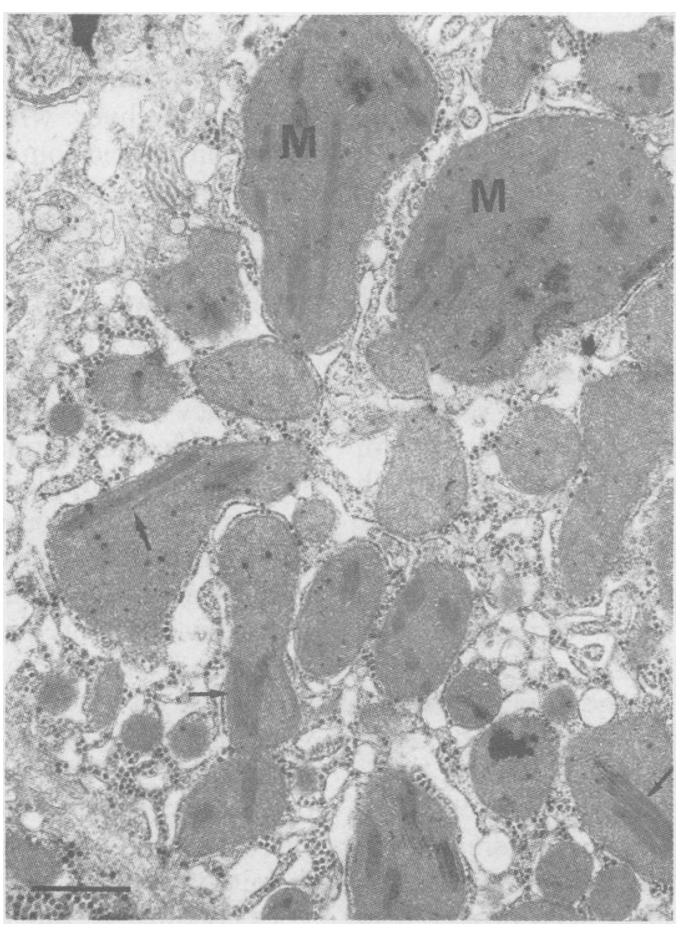

Figure 2: This electron micrograph illustrates the marked mitochondrial alterations present in the second liver specimen: pleiomorphism, numerous paracrystalline inclusions (arrows) and presence of megamitochondria $(M)$. (Electron microscopy;
lead citrate and uranyl acetate staining). Bar $=2 \mu \mathrm{m}$.
$0.5 \mathrm{mg} / \mathrm{l}$ (normal: 9-15) and serum vitamin A concentration was $550 \mathrm{mg} / \mathrm{l}$ (normal: $800-3000$ ). The patient was submitted to low fat diet with medium chain triglycerides $(2000 \mathrm{kcal}$ composed of $75 \mathrm{~g}$ protein, $80 \mathrm{~g}$ fat of which 30 were medium chain triglycerides, and $250 \mathrm{~g}$ carbohydrates) and received vitamins $E$ and $A$ supplementation ( $\alpha$-tocopheryl acetate, $1.5 \mathrm{~g}$ and vitamin $\mathrm{A}$, $15000 \mathrm{IU} /$ day). After one month, the administration of medium chain triglycerides was discontinued and the patient was left under vitamin supplementation alone.

In October 1989, at routine examination, a two-fold increase in serum concentrations of alanine aminotransferase was discovered. Other liver tests were normal. Serum markers for hepatitis B and C, cytomegalovirus and herpesvirus were absent. Antimitochondrial and antismooth muscle antibodies were absent. There was no evidence of alcohol consumption. No drug had been taken. A second liver biopsy was therefore taken on November 1989. Histological examination showed massive steatosis associated with liver fibrosis (Fig 1). Portal spaces were enlarged and fibrous tracts extended into the hepatic parenchyma. No annular fibrosis was observed. Enlarged portal tracts contained a moderate inflammatory infiltrate. Upon ultrastructural examination, the specimen disclosed a constellation of ultrastructural abnormalities associating: (a) marked hepatocytic changes, (b) signs of cytolysis, and (c) signs of liver fibrosis. In addition to the alterations present in the first liver specimen, marked mitochondrial changes were observed. In the majority of hepatocytes, large and bizarrely shaped mitochondria containing numerous paracrystalline inclusions were present (Fig 2). Typical megamitochondria could be found in some hepatocytes (Fig 2). Large oedematous mitochondria with clarified matrix and rare cristae were also observed. Peroxisomes, demonstrated by the catalase reaction, were shaped normally and were not increased in number. No Mallory body was observed. Signs of cytolysis were most often moderate, consisting in areas of focal cytoplasmic degeneration; in some instances, they were marked, including irregular nuclei, oedematous mitochondria and presence of autophagic vacuoles. In addition to hepatocytic alterations, sinusoidal changes were present. Deposits of perisinusoidal fibrosis could possibly be observed (Fig 3). In some instances, a basal membrane like material was present beneath the endothelial lining. Ito cells were neither hyperplastic nor hypertrophic. Some of them presented a transitional aspect, characterised by a fibroblastic appearance, with abundant endoplasmic reticulum and few lipid vacuoles.

Despite the supplementation, serum vitamin $E$ level was $1 \mathrm{mg} / \mathrm{l}$ and serum vitamin A level was $700 \mathrm{mg} / \mathrm{l}$. Simultaneous determination of hepatic levels of retinol and $\alpha$-tocopherol was performed using high performance liquid chromatography essentially as described by Bieri et $a l^{13}$ on liver homogenates. Two control samples from patients without lipoprotein disorders, obtained by liver biopsy for routine diagnostic purposes, were analysed in parallel. Hepatic concentration of vitamin A was markedly increased in our 


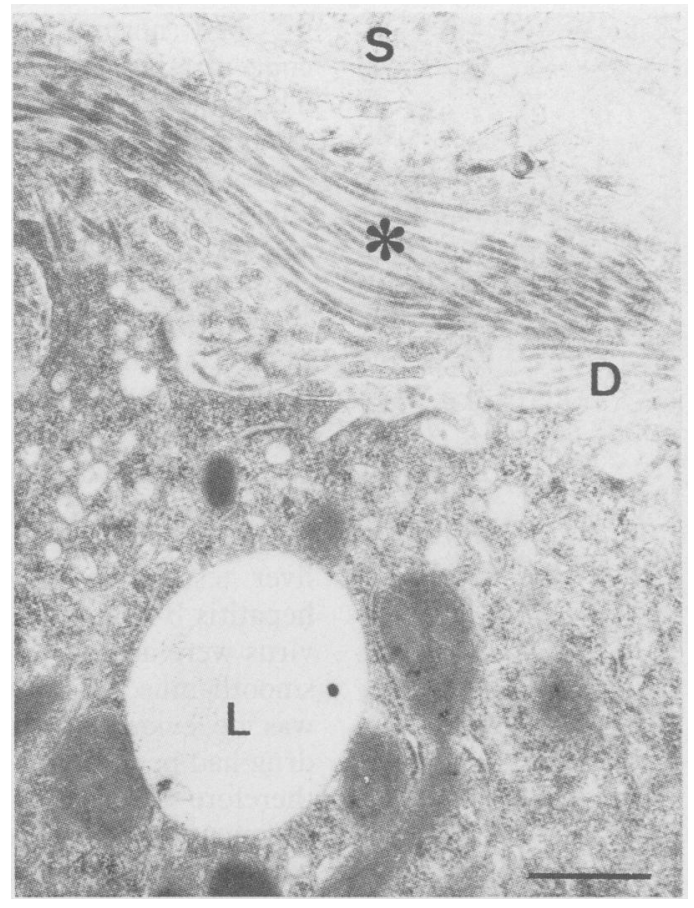

Figure 3: Perisinusoidal fibrosis is present in the form of collagen deposits $\left.{ }^{\star}\right)$ lying within the space of Disse $(D) . S$, sinusoidal lumen; L, lipid vacuole (Electron microscopy; lead citrate and uranyl acetate staining). Bar $=1 \mu \mathrm{m}$.

patient: $1100 \mu \mathrm{g} / \mathrm{g}$ of tissue, as compared with 92 and $243 \mu \mathrm{g} / \mathrm{g}$ respectively in the two controls. As regards the determination of $\alpha$-tocopherol, the variation between the two controls was too large to make it possible to interpret with confidence the slight increase detected in our patient.

\section{Discussion}

We report the case of a patient with homozygous familial hypobetalipoproteinaemia in whom liver fibrosis and hepatocytic alterations developed in the absence of long term administration of medium chain triglycerides.

The comparison of our morphological findings with those reported in abetalipoproteinaemia ${ }^{911115}$ shows that three types of morphological alterations of the liver might be observed in apoB-related disorders. A first type of lesion might be related to the abnormalities in lipoprotein synthesis, assembly and secretion: such alterations are steatosis, hypertrophy of Golgi apparatus and proliferation of smooth endoplasmic reticulum. ${ }^{15}$ Those changes are often asymptomatic and might be observed in the absence of other significant morphological alterations, as shown by the first liver specimen obtained from our patient and by other reports from the literature. ${ }^{15} \mathrm{~A}$ second type of change consists in signs of tissular and cellular damage, such as fibrosis, cytolysis, areas of focal cytoplasmic degeneration, and marked mitochondrial alterations, as observed in our patient and in some previous cases. ${ }^{911}$ Additional lesions, such as Mallory bodies, ${ }^{9}$ have also been observed in certain abetalipoproteinaemic patients. These changes are non-specific and bear strong similarities with those observed in other clinical situations, especially in alcoholic liver disease.
This makes it essential to rule out any intercurrent pathology by careful aetiological investigation. Finally, a possible specific ultrastructural lesion has been reported in one patient with abetalipoproteinaemia. " This lesion consisted in unusual alterations of organelles identified as peroxisomes, while not characterised by the catalase reaction. Despite the use of ultrastructural cytochemistry, we were unable to find comparable alterations in the present case. This is consistent with the fact that familial homozygous hypobetalipoproteinaemia and abetalipoproteinaemia have different molecular bases and constitute heterogeneous phenotypic categories. ${ }^{1-6}$

The relationship of hepatic lesions with the apoB-related disorder, if any, remains debatable. Their absence of specificity makes it difficult to assess their pathogenesis. It has been suggested that the medium chain triglycerides included in the low fat diet administered to abetalipoproteinaemic patients might have a toxic effect on hepatocytes. ${ }^{9}$ Our observation does not substantiate this hypothesis as our patient had not been subjected to long term administration of such compounds. We would raise an alternative hypothesis: that of an adverse effect of vitamin supplementation. This possibility is suggested by the increased level of vitamin A detected in the liver homogenates obtained from our patient. Hypervitaminosis A is well known to induce liver fibrosis and/or cirrhosis ${ }^{16-18}$ and might be accompanied by severe mitochondrial changes. ${ }^{18}$ It must be noted, however, that, in our patient, some characteristic lesions observed, such as hypertrophy and hyperplasia of perisinusoidal cells and pseudopeliotic sinusoidal dilatation, ${ }^{18-20}$ are lacking. Those lesions are not constant, however, especially at the stage of liver fibrosis. The risk of hepatic accumulation of lipid soluble vitamins, such as vitamins $A$ and $E$, in patients with apoB-related disorders is increased by the absence of formation of very low density lipoproteins by the liver, which results in an impaired secretion of lipid soluble vitamins. ${ }^{21}$

The possible occurrence of liver disease in the various apoB-related disorders is of clinical relevance. Liver tests must be included in the regular follow up of patients with such disorders. Attention must be paid to the quality of vitamin supplementation. On the one hand, vitamin deficiency might contribute to the aggravation of the neurological status, but on the other, excessive vitamin supplementation might lead to hepatic accumulation of potentially hepatotoxic compounds. A precise assessment of the vitaminic status of patients with apoB-related disorders is therefore important. It is not easy, however, to achieve, as serum concentrations of vitamin A do not parallel the tissue levels of this vitamin, as previously emphasised ${ }^{2223}$ and as illustrated by our patient. The only reliable method is direct tissue dosage, which must be therefore performed whenever a liver biopsy is required for diagnostic purposes.

In conclusion, our observation illustrates the possible occurrence of severe liver damage in familial homozygous hypobetalipoproteinaemia and underlines the importance of a regular follow up of liver function during the course of the 
disease. Accurate monitoring of vitamin supplementation must be achieved.

1 Infante R. Apolipoprotein B genetic deficiencies. In: Sirtori CR, Franceschini G, Brewer HB Jr, Assmann G, eds. Human apolipoprotein mutants. Vol 2. New York: Plenum Puman apolipoprotein mutants. Vol 2 .

2 Young SG. Recent progress in understanding apolipoprotein B. Circulation 1990; 82: 1574-94.

3 Cottrill C, Glueck CJ, Leuba V, Millett F, Puppione D, Brown WV. Familial homozygous hypobetalipoproteinemia. Metabolism 1973; 23: 779-91.

4 Ross RS, Gregg RE, Law SW, Monge SM, Grant SM, Higuchi $\mathrm{K}$, et al. Homozygous hypobetalipoproteinemia: a disease distinct from abetalipoproteinemia at the molecular level. $\mathcal{f}$ Clin Invest 1988; 81: 590-5.

5 Talmud PJ, Lloyd JK, Muller DPR, Collins DR, Scott J, Humphries S. Genetic evidence from two families that the apolipoprotein B gene is not involved in abetalipoproteinemia. $\mathcal{F}$ Clin Invest 1988; 82: 1803-6.

6 Bouma ME, Beucler I, Pessah M, Heinzmann C, Lusis AJ, Naim HY, et al. Description of two new patients with apobetalipoproteinemia: synthesis of a normal-sized apolipoapobetalipoproteinemia: synthesis of a normal-sized apolipoprotein 1 : 15 .

7 Muller DPR, Lloyd JK, Wolff OH. The role of vitamin E in the treatment of the neurological features of abetalipoproteinemia and other disorders of fat absorption. $\mathcal{F}$ Inherited Metab Dis 1985; 8 (suppl 1): 88-92.

8 Hegele RA, Angel A. Arrest of neuropathy and myopathy in abetalipoproteinemia with high-dose vitamin E therapy. Can Med Assoc F 1985; 132: 41-4.

9 Partin JS, Partin JC, Schubert WK, McAdams AJ. Liver ultrastructure in abetalipoproteinemia: evolution of micronodular cirrhosis. Gastroenterology 1974; 67: 107-18.

10 Illingworth DR, Connor WE, Miller RG. Abetalipoproteinemia. Report of two cases and review of therapy. Arch Neurol 1980; 37: 659-62.

11 Collins JC, Scheinberg JH, Giblin DR, Sternlieb I. Hepatic peroxisomal abnormalities in abetalipoproteinemia. Gastroenterology 1989; 97: 765-70.

12 Gay G, Pessah M, Bouma ME, Roche JF, Aymard JP, Beucler , Aggerbeck LP, Infante R. L'hypobetalipoprotéinémie familiale: etude familiale de 4 cas. Rev Med Interne 1990; 11: 273-9.

13 Bieri JG, Tolliver TJ, Catignani GL. Simultaneous determination of alpha-tocopherol and retinol in plasma cells or red cells by high pressure liquid chromatography. Am f Clin cells by high pressure
Nutr 1979; 32: 2143-9.

14 Willemin B, Coumaros D, Zerbe S, Weill-Bousson M, Annonier P, Hirsch E, et al. L'abetalipoprotéinémie. A propos de deux cas. Gastroenterol Clin Biol 1987; 11: 704-8.

15 Avigan MI, Ishak KM, Gregg RE, Hoofnagle JH. Morphologic features of the liver in abetalipoproteinemia. Hepatology 1984 ; 4 : $1233-6$.

16 Babb RR, Kieraldo JH. Cirrhosis due to hypervitaminosis A. West f Med 1978; 128: 244-6.

17 Muenter MD, Perry HO, Ludwig J. Chronic vitamin A intoxication in adults. Hepatic, neurologic and dermatologic complications. Am F Med 1971; 50: 129-36.

18 Phillips MJ, Poucell S, Patterson J, Valencia P. The liver. An atlas and text of ultrastructural pathology. New York: Raven Press, 1987.

19 Zafrani ES, Bernuau D, Feldmann G. Peliosis-like ultrastructural changes of the hepatic sinusoids in human chronic hypervitaminosis A: report of three cases. Hum Pathol 1984; 15: 1166-70.

20 Hruban Z, Russell RM, Boyer JL, Glagov S, Bagheri SA. Ultrastructural changes in liver of two patients with hypervitaminosis A. Am $\mathcal{F}$ Pathol 1974; 76: 451-61.

21 Cohn W, Loechleiter F, Weber F. $\alpha$-Tocopherol is secreted from rat liver in very low density lipoproteins. 7 Lipid Res 1988; 29: 1359-66.

22 Underwood BA, Siegel H, Weisell RC, Dolinski M. Liver stores of vitamin $A$ in a normal population dying suddenly or rapidly from unnatural causes in New York City. Am $\mathcal{F}$ Clin Nutr 1970; 23: 1037-42.

23 Bieri JG, Hoeg JM, Schaefer E, Zech LA, Brewer HB Jr. Vitamin $A$ and vitamin $E$ replacement in abetalipoproVitamin A and vitamin E replacement
teinemia. Ann Intern Med 1984; 100: 238-9. 